\title{
EXISTENCE THEOREMS ON UNBOUNDED SETS IN BANACH SPACES
}

\author{
NORIMICHI HIRANO AND WATARU TAKAHASHI
}

\begin{abstract}
ABSTRACr. In this paper, we give a necessary and sufficient condition under which a variational inequality defined on unbounded sets in a Banach space has a solution. Furthermore, we establish a necessary and sufficient condition under which the minimax equality on unbounded sets is true.
\end{abstract}

1. Introduction. Let $E$ be a real Banach space and $E^{*}$ its dual space. Let $T$ be a linear or nonlinear monotone operator from a closed convex set $C$ of $E$ into $E^{*}$. Then we say that $x_{0}$ satisfies a variational inequality if $x_{0} \in C$ and $\left(T x_{0}, x-x_{0}\right)$ $>0$ for all $x \in C$. This general form of a variational inequality has very interesting interpretations in various fields. For instance, when $C$ is a closed convex cone of $E$, then any solution $x_{0}$ of the variational inequality is actually a solution of the complementarity problem in mathematical programming, game theory and theory of economic equilibrium. For the theory of variational inequalities and the complementarity problem, we refer to the book by Barbu and Precupanu [2]. On the other hand, there are some generalizations of the famous minimax theorem of von Neumann [7]; for instance, see Barbu and Precupanu [2] and Takahashi [6].

In this paper, we obtain a necessary and sufficient condition under which a variational inequality on unbounded sets has a solution. Using this, we solve the complementarity problem and prove a fixed point theorem. Furthermore, we establish a necessary and sufficient condition under which the minimax equality on unbounded sets holds.

2. Variational inequalities. Let $E$ be a real reflexive Banach space and $C$ be a closed convex subset of $E$. A mapping $T: C \rightarrow E^{*}$ is said to be monotone if $(T x-T y, x-y)>0$ for all $x, y \in C$, and hemicontinuous on $C$ if for any $u, v \in C$, the mapping $t \rightarrow T(t v+(1-t) u)$ of $[0,1]$ to $E^{*}$ is continuous when $E^{*}$ is endowed with the weak ${ }^{*}$ topology. Also $T$ is said to be coercive on $C$ if for some $u \in C$,

$$
\lim _{\|x\| \rightarrow \infty ; x \in C}(T x, x-u) /\|x\|=+\infty .
$$

A mapping $F: C \rightarrow E$ is said to be nonexpansive if for any $x, y \in C,\|F x-F y\|$ $<\|x-y\|$. We note that if $E$ is a real Hilbert space and $F: C \rightarrow E$ is nonexpansive, then $I-F$ is a monotone mapping of $C$ into $E$. Let $H, K$ be nonempty closed subsets of the Banach space $E$; then we denote by $\partial_{H} K$ the set of $z \in K$ such that $U(z) \cap(H-K) \neq \varnothing$ for every neighborhood $U(z)$ of $z$ and by $i_{H} K$ the set of $z \in K$ such that $U(z) \cap(H-K)=\varnothing$ for some neighborhood $U(z)$ of $z$.

Received by the editors June 5, 1979.

1980 Mathematics Subject Classification. Primary 47H0S, 47H10, 49A40. 
ThEOREM 1. Let $C$ be a nonempty closed convex subset of a reflexive Banach space $E$ and $T$ be a monotone and hemicontinuous mapping of $C$ into $E^{*}$. Then the following conditions are equivalent.

(1) There exists $x_{0} \in C$ such that $\left(T x_{0}, y-x_{0}\right)>0$ for all $y \in C$;

(2) there exists a bounded closed convex subset $K$ of $C$ such that for each $z \in \partial_{C} K$, there exists $y \in i_{C} K$ which satisfies $(T z, y-z)<0$.

Proof. First we show that (1) implies (2). Let $x_{0}$ be an element of $C$ such that $\left(T x_{0}, y-x_{0}\right)>0$ for all $y \in C$. Set $d=\left\|x_{0}-y_{0}\right\|$ where $y_{0} \in C$ and $y_{0} \neq x_{0}$, and $K=\left\{x \in C:\left\|x-x_{0}\right\|<d\right\}$. Then we have $x_{0} \in i_{C} K$. Let $z \in \partial_{C} K$. By the monotonicity of $T$, it follows that $\left(T z, z-x_{0}\right)>\left(T x_{0}, z-x_{0}\right)>0$. Therefore, we have $\left(T z, x_{0}-z\right)<0$. Next we show that (2) implies (1). Let $K$ be a bounded closed convex subset of $C$ which satisfies the condition (2). Since $K$ is weakly compact convex, there exists $x_{0} \in K$ such that $\left(T x_{0}, x-x_{0}\right)>0$ for all $x \in K$ (cf. [2], [3]). If $x_{0} \in i_{C} K$, then for each $y \in C$ we can choose $\lambda>0$ so small that $x=\lambda y+(1-\lambda) x_{0}$ lies in $K$. Then $\left(T x_{0}, \lambda y+(1-\lambda) x_{0}-x_{0}\right)>0$ and hence $\lambda\left(T x_{0}, y-x_{0}\right)>0$. Cancelling $\lambda$, we have $\left(T x_{0}, y-z_{0}\right)>0$. If $x_{0} \in \partial_{C} K$, then, by the hypothesis, there exists $z_{0} \in i_{C} K$ such that $\left(T x_{0}, z_{0}-x_{0}\right)<0$. Since $\left(T x_{0}, x-\right.$ $\left.x_{0}\right)>0$ for all $x \in K$, we have $\left(T x_{0}, x-z_{0}\right)>0$ for all $x \in C$. Since $z_{0} \in i_{C} K$, for each $y \in C$, there exists $\lambda>0$ such that $x=\lambda y+(1-\lambda) z_{0}$ lies in $K$. Then $\lambda\left(T x_{0}, y-z_{0}\right)>0$. Cancelling $\lambda$, we have $\left(T x_{0}, y-z_{0}\right)>0$. Then since $\left(T x_{0}, z_{0}\right.$ $\left.-x_{0}\right)>0$, we obtain $\left(T x_{0}, y-x_{0}\right)>0$.

The following corollaries are direct consequences of Theorem 1.

Corollary 1 (CF. [3]). Let $C$ be a nonempty closed convex subset of a reflexive Banach space $E$ and $T$ be a monotone hemicontinuous mapping of $C$ into $E^{*}$. If $T$ is coercive on $C$, then there exists $x_{0} \in C$ such that $\left(T x_{0}, y-x_{0}\right)>0$ for all $y \in C$.

Proof. It is sufficient to show that the coercivity condition implies the condition (2) of Theorem 1. By the definition of coercivity, there exist $y \in C$ and positive numbers $c, k$ such that $\|y\|<c$ and $(T x, x-y)>k\|x\|$ for $x \in C$ with $\|x\|>c$. If we set $K=\{x \in C:\|x\|<c\}$, then it is obvious that $K$ satisfies the condition (2) of Theorem 1.

Corollary 1 has a very interesting interpretation when $C$ is a closed convex cone.

CoROllaRy 2. Let $C$ be a nonempty closed convex cone in a reflexive Banach space $E$ and $T$ be a monotone hemicontinuous mapping of $C$ into $E^{*}$. If $T$ is coercive, then there exists an $x_{0} \in C$ such that $-T x_{0} \in C^{*}$ and $\left(T x_{0}, x_{0}\right)=0$ where $C^{*}=\{u$ $\in E^{*}:(u, x)<0$ for all $\left.x \in C\right\}$.

Proof. By Corollary 1 , there exists $x_{0} \in C$ such that $\left(T x_{0}, y-x_{0}\right)>0$ for all $y \in C$. It follows from Lemma 3.1 of [4] that $-T x_{0} \in C^{*}$ and $\left(T x_{0}, x_{0}\right)=0$.

COROLlary 3. Let $C$ be a nonempty closed convex subset of a Hilbert space $H$ such that $0 \in C$ and $T$ be a nonexpansive mapping of $C$ into $H$. If there exists $a$ bounded closed convex set $K \subset C$ such that $0 \in i_{C} K$ and $\|T z\|<\|z\|$ for all $z \in \partial_{C} K$, then there exists an $x_{0} \in C$ such that $\left\|x_{0}-T x_{0}\right\|=\min \left\{\left\|y-T x_{0}\right\|: y \in C\right\}$. Particularly, if $T$ maps $C$ into itself, there exists $x_{0} \in C$ such that $T x_{0}=x_{0}$. 
Proof. It is obvious that the mapping $I-T$ of $C$ into $H$ is monotone and hemicontinuous. Since $\|T z\|<\|z\|$ for all $z \in \partial_{C} K$, we have $(z-T z,-z)<0$ for all $z \in \partial_{C} K$. Since $0 \in i_{C} K, K$ satisfies the condition (2) of Theorem 1. Therefore there exists $x_{0} \in C$ such that $\left(x_{0}-T x_{0}, y-x_{0}\right)>0$ for all $y \in C$. Hence we obtain $\left\|x_{0}-T x_{0}\right\|<\left\|y-T x_{0}\right\|$ for all $y \in C$. Particularly, if $T$ maps $C$ into itself, we have $\min \left\{\left\|y-T x_{0}\right\|: y \in C\right\}=0$ and hence $T x_{0}=x_{0}$.

3. Minimax theorem. Next we consider a minimax theorem and establish a necessary and sufficient condition under which the minimax equality on unbounded sets holds.

Theorem 2. Let $X, Y$ be reflexive Banach spaces, and let $A \subset X, B \subset Y$ be nonempty closed convex sets. If $F$ is a function on $A \times B$ such that for each $y \in B$, $F(\cdot, y)$ is an upper semicontinuous concave function on $A$ and for each $x \in A, F(x, \cdot)$ is a lower semicontinuous convex function on $B$, then the following conditions are equivalent.

(1)

$$
\max _{x \in A} \min _{y \in B} F(x, y)=\min _{y \in B} \max _{x \in A} F(x, y)
$$

(2) there exist bounded closed convex sets $K \subset A$ and $L \subset B$ such that for each $(x, y) \in\left(\partial_{A} K \times L\right) \cup\left(K \times \partial_{B} L\right)$, there exists $a(u, v) \in i_{A} K \times i_{B} L$ which satisfies $F(u, y)>F(x, v)$.

Proof. First we show that (1) implies (2). If (1) holds, then there exists $\left(x_{0}, y_{0}\right) \in A \times B$ such that $F\left(x_{0}, y\right)>F\left(x_{0}, y_{0}\right)>F\left(x, y_{0}\right)$ for all $(x, y) \in A \times B$. Let $K=\left\{x \in A:\left\|x_{0}-x\right\|<\left\|x_{0}-a\right\|\right\}$ and $L=\left\{y \in B:\left\|y_{0}-y\right\|<\| y_{0}-\right.$ $b \|\}$, where $a \in A, b \in B, x_{0} \neq a$ and $y_{0} \neq b$. Then we have $\left(x_{0}, y_{0}\right) \in i_{A} K \times i_{B} L$ and $F\left(x_{0}, y\right)>F\left(x_{0}, y_{0}\right) \geqslant F\left(x, y_{0}\right)$ for all $(x, y) \in\left(\partial_{A} K \times L\right) \cup\left(K \times \partial_{B} L\right)$. Next we show that (2) implies (1). Let $K$ and $L$ be bounded closed convex sets which satisfy the condition (2). Then, by Theorem 3.8 of [2], there exists $\left(x_{0}, y_{0}\right) \in K \times L$ such that $F\left(x, y_{0}\right)<F\left(x_{0}, y_{0}\right)<F\left(x_{0}, y\right)$ for all $(x, y) \in K \times L$. Let $\left(x_{0}, y_{0}\right) \in i_{A} K$ $\times i_{B} L$. Then for each $x \in A$ we can choose $\lambda>0$ so small that $\lambda x+(1-\lambda) x_{0} \in$ $K$. Since $F(\cdot, y)$ is concave, we have

$$
F\left(x_{0}, y_{0}\right)>F\left(\lambda x+(1-\lambda) x_{0}, y_{0}\right)>\lambda F\left(x, y_{0}\right)+(1-\lambda) F\left(x_{0}, y_{0}\right)
$$

and hence $F\left(x, y_{0}\right)<F\left(x_{0}, y_{0}\right)$. Also we obtain that $F\left(x_{0}, y_{0}\right)<F\left(x_{0}, y\right)$ for all $y \in B$. So, (1) holds. Let $\left(x_{0}, y_{0}\right) \in\left(\partial_{A} K \times L\right) \cup\left(K \times \partial_{B} L\right)$. Then by the condition (2) there exists $(u, v) \in i_{A} K \times i_{B} L$ such that $F\left(u, y_{0}\right)>F\left(x_{0}, v\right)$. Since $F\left(x, y_{0}\right)<F\left(x_{0}, y_{0}\right)<F\left(x_{0}, y\right)$ for all $(x, y) \in K \times L$, we have $F\left(u, y_{0}\right)=$ $F\left(x_{0}, y_{0}\right)=F\left(x_{0}, v\right)$. For each $x \in A$, we take $\lambda>0$ so small that $\lambda x+(1-\lambda) u$ $\in K$. Then

$$
\begin{aligned}
F\left(x_{0}, y_{0}\right) & >F\left(\lambda x+(1-\lambda) u, y_{0}\right) \\
& >\lambda F\left(x, y_{0}\right)+(1-\lambda) F\left(u, y_{0}\right) \\
& =\lambda F\left(x, y_{0}\right)+(1-\lambda) F\left(x_{0}, y_{0}\right) .
\end{aligned}
$$

Hence we obtain that $F\left(x, y_{0}\right)<F\left(x_{0}, y_{0}\right)$. Also we obtain that $F\left(x_{0}, y_{0}\right)<F\left(x_{0}, y\right)$ for all $y \in B$. This completes the proof. 
Corollary 4 (CF. [2]). Let $X, Y, A, B$ and $F$ satisfy the assumptions as in Theorem 2. If there exists $\left(x_{0}, y_{0}\right) \in A \times B$ such that

$$
\lim _{\substack{\|x\|+\|y\| \rightarrow \infty \\(x, y) \in A \times B}}\left\{F\left(x_{0}, y\right)-F\left(x, y_{0}\right)\right\}=\infty,
$$

then we have $\max _{x \in A} \min _{y \in B} F(x, y)=\min _{y \in B} \max _{x \in A} F(x, y)$.

Proof. It is clear from the hypothesis that there exists $k>0$ such that $\left\|x_{0}\right\|<k$, $\left\|y_{0}\right\|<k$ and for every $(x, y) \in A \times B$ with $\|x\|+\|y\|>k$ we have $F\left(x_{0}, y\right)-$ $F\left(x, y_{0}\right)>0$. Let $K=\{x \in A:\|x\|<k\}$ and $L=\{y \in B:\|y\|<k\}$. Then for every $(x, y) \in\left(\partial_{A} K \times L\right) \cup\left(K \times \partial_{B} L\right)$, we obtain $F\left(x_{0}, y\right)>F\left(x, y_{0}\right)$. So, we obtain Corollary 4 from Theorem 2.

\section{REFERENCES}

1. M. S. Bazaraa, J. Goode and M. S. Nashed, A nonlinear complementarity problem in mathematical programming in Banach spaces, Proc. Amer. Math. Soc. 35 (1972), 165-170.

2. V. Barbu and Th. Precupanu, Convexity and optimization in Banach spaces, Editura Academiei R.S.R., Bucharest, 1975.

3. H. Brézis, Equations et inéquations non linéaires dans les espaces vectoriels en dualité, Ann. Inst. Fourier (Grenoble) 18 (1965), 115-175.

4. S. Karamardian, Generalized complementarity problem, J. Optimization Theory Appl. 8 (1971), 161-168.

5. J. J. Moré, Coercioity conditions in nonlinear complementarity problems, SIAM Rev. 16 (1974), 1-16.

6. W. Takahashi, Nonlinear variational inequalities and fixed point theorems, J. Math. Soc. Japan 28 (1976), 168-181.

7. J. von Neumann, Zür theorie der gesellshaft spiele, Math. Ann. 100 (1928), 295-320.

Department of Information Sciences, Tokyo Institute of Tbchnology, Oh-orayama, MrguroKU, TOKYO, JAPAN, 152 